\title{
The mutation causing the black-and-tan pigmentation phenotype of Mangalitza pigs maps to the porcine ASIP locus but does not affect its coding sequence
}

\author{
Cord Drögemüller, ${ }^{1,2}$ Alexander Giese, ${ }^{1}$ Flávia Martins-Wess, ${ }^{1}$ Sabine Wiedemann, ${ }^{3}$ \\ Leif Andersson, ${ }^{4}$ Bertram Brenig, ${ }^{5}$ Ruedi Fries, ${ }^{3}$ Tosso Leeb ${ }^{1,2}$ \\ ${ }^{1}$ Institute of Animal Breeding and Genetics, University of Veterinary Medicine Hannover, 30559 Hannover, Germany \\ ${ }^{2}$ Institute of Genetics, Vetsuisse Faculty, University of Berne, 3012 Berne, Switzerland \\ ${ }^{3}$ Chair of Animal Breeding and Molecular Genetics, Technical University of Munich, 85354 Freising-Weihenstephan, Germany \\ ${ }^{4}$ Swedish University of Agricultural Sciences, Uppsala, Sweden \\ ${ }^{5}$ Institute of Veterinary Medicine, University of Göttingen, 37073 Göttingen, Germany
}

Received: 29 July 2005 / Accepted: 13 September 2005

\begin{abstract}
The gene for agouti signaling protein (ASIP) is centrally involved in the expression of coat color traits in animals. The Mangalitza pig breed is characterized by a black-and-tan phenotype with black dorsal pigmentation and yellow or white ventral pigmentation. We investigated a Mangalitza $\times$ Piétrain cross and observed a coat color segregation pattern in the $\mathrm{F}_{2}$ generation that can be explained by virtue of two alleles at the $M C 1 R$ locus and two alleles at the ASIP locus. Complete linkage of the black-and-tan phenotype to microsatellite alleles at the ASIP locus on SSC 17q21 was observed. Corroborated by the knowledge of similar mouse coat color mutants, it seems therefore conceivable that the black-and-tan pigmentation of Mangalitza pigs is caused by an ASIP allele $a^{t}$, which is recessive to the wild-type allele $A$. Toward positional cloning of the $a^{t}$ mutation, a 200-kb genomic BAC/PAC contig of this chromosomal region has been constructed and subsequently sequenced. Full-length ASIP cDNAs obtained by RACE differed in their $5^{\prime}$ untranslated regions, whereas they shared a common open reading frame. Comparative sequencing of all ASIP exons and ASIP cDNAs between Mangalitza and Piétrain pigs did not reveal any differences associated with
\end{abstract}

Nucleotide sequence data reported are available in the EMBL database under accession numbers AJ427478, AJ634673AJ634677, and AM050718.

Correspondence to: Tosso Leeb, Institute of Genetics, Vetsuisse Faculty University of Berne, Bremgartenstr. 109a, 3012 Berne, Switzerland; E-mail: Tosso.Leeb@itz.unibe.ch the coat color phenotype. Relative qRT-PCR analyses showed different dorsoventral skin expression intensities of the five ASIP transcripts in black-andtan Mangalitza. The $a^{t}$ mutation is therefore probably a regulatory ASIP mutation that alters its dorsoventral expression pattern.

\section{Introduction}

Agouti signaling protein (ASIP) acts as an antagonist to $\alpha$-melanocortin stimulating hormone $(\alpha-\mathrm{MSH})$ at the melanocortin receptor 1 (MC1R) (Lu et al. 1994). $\mathrm{MC} 1 \mathrm{R}$ activation, following binding of $\alpha-\mathrm{MSH}$, leads to the production of black eumelanin in hair follicles. In contrast, the binding of the high-affinity antagonist ASIP blocks MC1R signaling and leads to the production of red pheomelanin. The $M C 1 R$ gene corresponds to the classical Extension $(E)$ coat color locus in mammals. In addition to binding to the MC1R, ASIP also acts as antagonist at the melanocortin receptor 4 (MC4R), where it regulates food intake and lipid metabolism (Harris et al. 2002). Inhibition of the MC4R by ectopically expressed high levels of ASIP in the agouti lethal yellow mouse leads to pronounced obesity (Carroll et al. 2004). A similar obese phenotype results from the targeted disruption of the $M c 4 r$ gene in knockout mice (Huszar et al. 1997). Therefore, the ASIP gene can be considered a candidate locus for feed intake and fat deposition in pigs.

The ASIP locus is central in the expression of coat color traits in animals. In mice, genetic analyses 

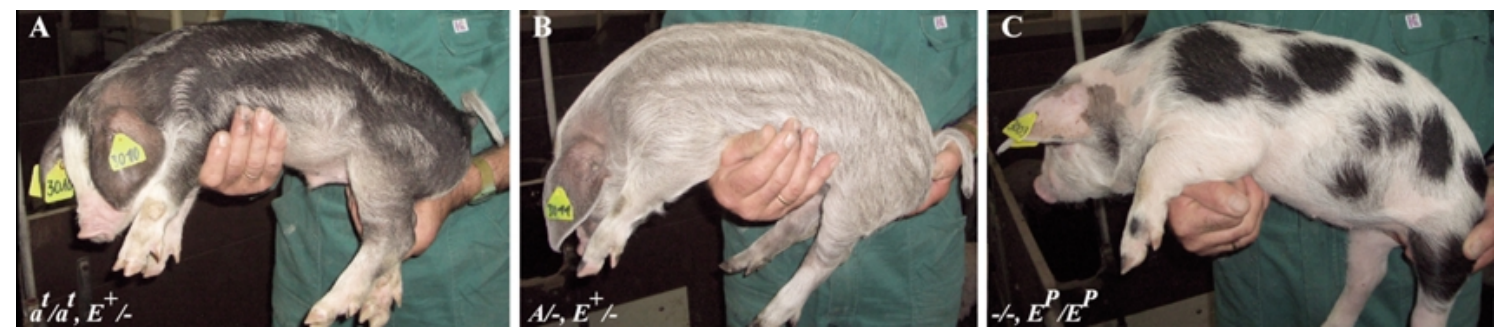

Fig. 1. Coat color phenotypes in the resource family. (A) Black-and-tan $F_{2}$ piglet. The stripes were visible only in juvenile animals; they disappeared in adult animals. (B) Gray $\mathrm{F}_{2}$ piglet. Similar to the black-and-tan animals, the stripes of these animals also vanished in adult animals. (C) Black-spotted $\mathrm{F}_{2}$ piglet. The hypothesized $A S I P$ and $M C 1 R$ genotypes for the three different phenotypes are indicated.

showed that Mcr1 alleles are epistatic to Asip alleles (Siracusa 1994). Among the variety of coat color phenotypes in the domestic pig, only two breeds, Duroc and Mangalitza, are characterized by general or regional pheomelanin expression. While porcine $M C 1 R$ alleles have been previously characterized at the molecular level (Kijas et al. 1998b, 2001), so far no functional mutation of the porcine ASIP gene has been reported. In pigs a recessively inherited $M C 1 R$ loss-of-function mutation that is responsible for generalized pheomelanin production has been identified in the red-colored Duroc breed (Kijas et al. 1998b). The "swallow-bellied" Mangalitza pig breed is characterized by black dorsal pigmentation and yellow or white ventral pigmentation and a sharp lateral line of demarcation. Similar phenotypes in black-and-tan or white-bellied agouti mice are associated with the $a^{t}$ or $A^{W}$ alleles at the Asip locus, which are recessive to the wild-type allele A. The $a^{t}$ and $A^{W}$ alleles in the mouse arose from $6-\mathrm{kb}$ or 0.6 $\mathrm{kb}$ insertions, respectively, into important regulatory regions of the murine Asip intron 1 (Bultman et al. 1994). It still remains a fundamental question in developmental biology how adjacent regions of the vertebrate body acquire differences in their appearance or morphology.

ASIP cDNAs have been cloned from several mammalian species including human, mouse, cattle, dog, and fox (Bultman et al. 1992; Girardot et al. 2005; Kerns et al. 2004; Kwon et al. 1994; Vage et al. 1997). In all analyzed mammalian species the ASIP cDNA is approximately $700 \mathrm{bp}$ long and encodes a protein of 131-133 amino acids. The murine Asip gene consists of three coding exons termed exons 2-4 and four $5^{\prime}$-untranslated exons termed exon 1A, exon 1A', exon 1B, and exon 1C, respectively (Bultman et al. 1992). Contiguous porcine genomic sequences of the 5'-untranslated exons, which are separated by large introns from the coding part of the gene, have not been available in the public databases so far. The chromosomal location of the porcine ASIP gene has been assigned to SSC 17q21 (Kijas et al. 1998a). We have previously characterized the genomic organization and SNPs of the coding exons of the porcine ASIP gene as well as the downstream flanking gene $A H C Y$ (Leeb et al. 2000; Leeb and Rohrer 2002).

The initial aim of the study was to identify the gene underlying the black-and-tan phenotype in pigs using a Mangalitza $\times$ Piétrain $\mathrm{F}_{2}$ resource population. In this article we report complete linkage between ASIP and this phenotype. We also provide an extended analysis of the genomic organization of the porcine ASIP locus. A mutation scan of the complete exonic and partial intronic $A S I P$ sequence did not uncover the causative mutation for the porcine $a^{t}$ allele. However, expression analyses of five different ASIP transcripts in black-and-tan Mangalitza showed differences in dorsal and ventral expression and suggested the existence of a regulatory ASIP mutation.

\section{Material and methods}

Resource population construction. Two Mangalitza boars were mated to 20 Piétrain sows establishing an $F_{1}$ generation consisting of 7 boars and 37 sows. $F_{1}$ intercross resulted in 1492 piglets. Coat color phenotypes of all animals were classified as black-andtan, gray, or black-spotted, respectively (Fig. 1; Wiedemann et al. 2000). DNA samples of all founder animals, $76 \quad F_{2}$ black-and-tan piglets, and their $F_{1}$ parents were used for genotyping. The Mangalitza founders were postulated to be homozygous for the $A S I P^{*} a^{t}$ allele and homozygous for the wild-type $M C 1 R^{*} E^{+}$allele, respectively. The Piétrain founders were assumed to be homozygous for the wild-type $A S I P^{*} A$ allele and for the breed-specific $M C 1 R^{*} E^{P}$ allele (Kijas et al. 1998b).

Microsatellite genotyping. A polymorphic dinucleotide repeat $(\mathrm{AC})_{21}$ denoted $A g C A$ was derived from a porcine genomic BAC clone containing the porcine ASIP gene (Kijas et al. 1998a). The 
primers AgCA-F (5'-CCC TTA GGT GGA AAT TAG CTA GAG-3') and AgCA-R (5'-CTC ATA TCA CCC CTG AGA GGT AG-3') were used for microsatellite fragment analyses in the resource population using an ABI 377 sequencer with Genescan 3.1 and Genotyper 2.5 software (Applied Biosystems, Foster City, CA) (Wiedemann et al. 2000). In addition, linkage mapping of the ASIP gene-associated microsatellite $A g C A$ was performed within the Wild Boar $\times$ Large White pedigree (Ellegren et al. 1994). PCR was carried out in a total volume of $20 \mu \mathrm{l}$ containing $25 \mathrm{ng}$ genomic DNA, $1.0 \mathrm{mM} \mathrm{MgCl}_{2}, 50 \mathrm{mM} \mathrm{KCl}, 10 \mathrm{mM}$ Tris-HCl, pH 8.3, 200 pM dNTPs, 0.5 U AmpliTaq Gold (Applied Biosystems, Darmstadt, Germany), and 10 pmol of both AgCA1F and AgCA1R. Initial heat denaturation was carried out at $94^{\circ} \mathrm{C}$ for $10 \mathrm{~min}$ followed by 32 cycles each consisting of $45 \mathrm{sec}$ at $94^{\circ} \mathrm{C}, 45 \mathrm{sec}$ at $55^{\circ} \mathrm{C}$, and $45 \mathrm{sec}$ at $72^{\circ} \mathrm{C}$.

Linkage and haplotype analysis. The evaluation of the data using the MERLIN software was based on a multipoint analysis, including identicalby-descent calculations, kinship calculations, and nonparametric linkage analysis (Abecasis et al. 2002). The generated genotype information of the families was tested for linkage using the $n p l$ scoring function. Moreover, we estimated haplotypes by finding the most likely path of gene flow. To reconstruct likely haplotypes and identify recombination events we applied the best option of MERLIN.

DNA library screening. Library screenings were done as described (Martins-Wess et al. 2002). Briefly, the TAIGP714 PAC library (Al-Bayati et al. 1999; http://www.rzpd.de) was screened by PCR of hierarchical DNA pools. This library has been constructed from a male German Landrace pig; thus, it was assumed that clones from this library should represent wild-type $A S I P^{*} A$ alleles. The porcine genomic BAC library RPCI-44 was screened according to the RPCI (Roswell Park Cancer Institute) protocols (http:// www.chori.org/bacpac/). This library has been constructed using DNA from four male pigs (breed: $37.5 \%$ Yorkshire, $37.5 \%$ Landrace, and 25\% Meishan); thus, it was assumed that clones from this library also represent wild-type $A S I P^{*} A$ alleles.

DNA sequence analysis. End sequences of isolated BAC and PAC DNA were generated with a LICOR 4200 automated sequencer system. Further analyses were performed with the online tools of the European Bioinformatics Institute (http://www. ebi.ac.uk/), BLAST database searches in the GenBank database of the National Center for Biotechnology Information (NCBI) (http://www.ncbi.nlm. nih.gov/l and the RepeatMasker searching tool for repetitive elements (A.F.A. Smit and P. Green, http://repeatmasker.genome.washington.edu//).

Single copy sequences were used to design primer pairs for the chromosome walking using the programs GeneFisher and Primer3 (http://bibiserv.techfak.unibielefeld.de/cgi-bin/gf_submit?mode=START and http://www-genome.wi.mit.edu/cgi-bin/primer/prim er3_www.cgi). PAC and BAC DNA was restricted with different enzymes and separated on $0.8 \%$ agarose gels. DNA fragments were excised and cloned into the polylinker of pGEM-4Z (Promega, Mannheim, Germany). Recombinant plasmid DNA was sequenced with the ThermoSequenase kit (GE Healthcare, Freiburg, Germany) and a LICOR 4200 automated sequencer. After sequencing a collection of plasmid subclones, remaining gaps were closed by a primer walking strategy until both strands were completely sequenced. Sequence data were analyzed with Sequencher 4.2 (GeneCodes, Ann Arbor, MI).

Mutation analysis. To identify variations within the porcine ASIP sequence, exons with flanking regions were PCR amplified from 13 individuals of a single three-generation pedigree of the established resource population (Fig. 2). PCR primers and conditions for the amplification of ASIP exons with flanking sequences and partial intronic sequences are shown in Supplementary Table 1. The PCR products were directly sequenced using the PCR primers or internal sequencing primers on a MegaBace1000 sequencer (GE Healthcare, Freiburg, Germany).

cDNA sequence analysis. The isolation of fulllength cDNAs was achieved by a modified rapid amplification of cDNA ends (RACE) protocol. The porcine-coding mRNA sequences were used for RACE primer design. Total RNA from the skin of a pig was used for the 5'-RACE PCR products with the FirstChoice $^{\mathrm{TM}}$ RNA ligase-mediated (RLM)-RACE kit (Ambion Europe, Huntingdon, UK) according to the instructions of the manufacturer. After cloning and sequencing of RACE PCR products, the exact porcine ASIP genomic structure was determined using the mRNA-to-genomic alignment program Spidey (http://www.ncbi.nlm.nih.gov/IEB/Research/ Ostell/Spidey/index.html).

RNA isolation and quantitative RT-PCR. Ventral and dorsal skin samples from a swallow-bellied Mangalitza sow and from the black region of a belted Hampshire sow were isolated directly after biopsy. The RNA was isolated with the RNeasy 96 Universal Tissue kit (Qiagen, Hilden, Germany) according to 


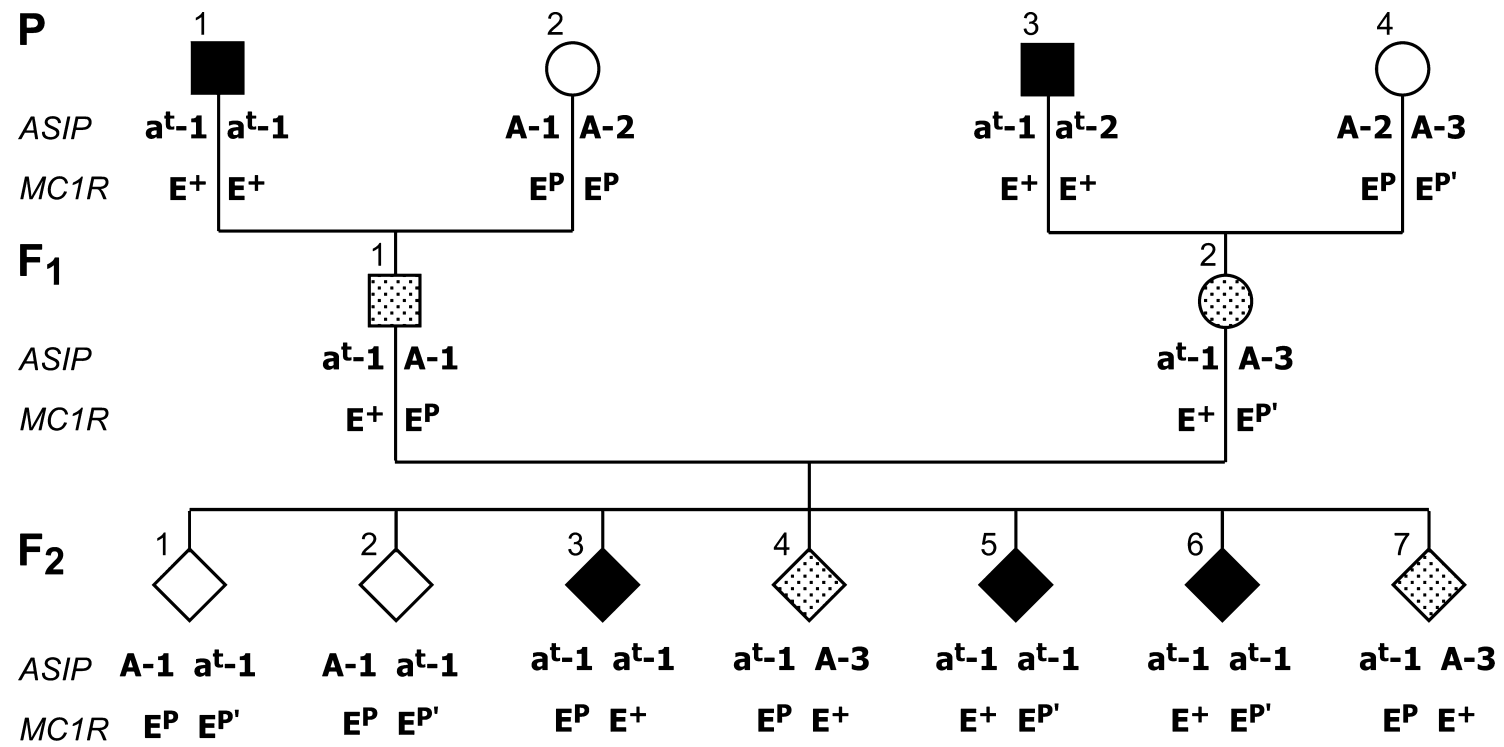

Fig. 2. Three-generation $F_{2}$ pedigree of a single family from the Mangalitza $\times$ Piétrain resource population. Filled symbols indicate animals with black-and-tan phenotype, shaded symbols the gray phenotype, and open symbols the black-spotted phenotype. Beneath the symbols the ASIP and MC1R genotypes of the animals are indicated. The ASIP genotypes correspond to the haplotypes as described in Table 1.

Table 1. Nucleotide polymorphisms within the porcine ASIP region and deduced haplotypes for genotyped markers in the subfamily shown in Fig. 2

\begin{tabular}{|c|c|c|c|c|c|c|c|}
\hline \multirow[b]{3}{*}{ Type of polymorphism } & \multirow[b]{3}{*}{ Position $^{\mathrm{a}}$} & \multirow[b]{3}{*}{ A/427478 } & \multicolumn{5}{|c|}{ Haplotypes } \\
\hline & & & \multicolumn{2}{|c|}{ Mangalitza } & \multicolumn{3}{|c|}{ Piétrain } \\
\hline & & & $a^{t}-1$ & $a^{t}-2$ & $A-1$ & $A-2$ & $A-3$ \\
\hline $\mathrm{C} / \mathrm{T}$ SNP & 1544 & $\mathrm{C}$ & $\mathrm{T}$ & $\mathrm{C}$ & $\mathrm{T}$ & $\mathrm{T}$ & $\mathrm{C}$ \\
\hline A/G SNP & 4818 & A & A & G & A & A & G \\
\hline A/T SNP & 9804 & A & A & $\mathrm{T}$ & A & A & $\mathrm{T}$ \\
\hline C/T SNP & 15188 & $\mathrm{C}$ & $\mathrm{C}$ & $\mathrm{T}$ & $\mathrm{C}$ & $\mathrm{C}$ & $\mathrm{T}$ \\
\hline A/T SNP & 20386 & A & A & $\mathrm{A}$ & A & A & $\mathrm{A}$ \\
\hline 1 bp InDel & 25904 & G & del & G & G & del & G \\
\hline A/G SNP & 31334 & A & A & G & A & A & G \\
\hline A/G SNP & 36717 & G & G & $\mathrm{A}$ & $\mathrm{G}$ & G & A \\
\hline $\mathrm{A} / \mathrm{G}$ SNP & 41369 & A & A & A & $\mathrm{A}$ & $\mathrm{A}$ & A \\
\hline $\mathrm{C} / \mathrm{T}$ SNP & 46419 & $\mathrm{C}$ & $\mathrm{C}$ & $\mathrm{T}$ & $\mathrm{C}$ & $\mathrm{C}$ & $\mathrm{T}$ \\
\hline C/T SNP & 52426 & $\mathrm{C}$ & $\mathrm{T}$ & $\mathrm{C}$ & $\mathrm{C}$ & $\mathrm{C}$ & $\mathrm{C}$ \\
\hline 29 bp InDel & 63140 & ins & del & ins & ins & del & del \\
\hline G/T SNP & 69000 & G & G & G & $\mathrm{G}$ & $\mathrm{G}$ & $\mathrm{G}$ \\
\hline $\mathrm{G} / \mathrm{T}$ SNP & 82586 & G & $\mathrm{G}$ & $\mathrm{T}$ & $\mathrm{G}$ & G & $\mathrm{G}$ \\
\hline C/T SNP & 85626 & $\mathrm{~T}$ & $\mathrm{~T}$ & $\mathrm{C}$ & $\mathrm{T}$ & $\mathrm{T}$ & $\mathrm{C}$ \\
\hline G/A SNP & 86420 & $\mathrm{G}$ & $\mathrm{A}$ & G & $\mathrm{G}$ & G & $\mathrm{G}$ \\
\hline G/A SNP & 107736 & G & G & $\mathrm{A}$ & G & G & A \\
\hline A/G SNP & 116324 & A & $\mathrm{G}$ & A & $\mathrm{A}$ & $\mathrm{A}$ & G \\
\hline G/T SNP & 126603 & G & $\mathrm{T}$ & G & G & G & G \\
\hline G/A SNP & 133012 & G & G & A & G & G & G \\
\hline C/T SNP & 135533 & $\mathrm{~T}$ & $\mathrm{C}$ & $\mathrm{T}$ & $\mathrm{T}$ & $\mathrm{T}$ & $\mathrm{T}$ \\
\hline A/G SNP & 162900 & $\mathrm{~A}$ & A & G & A & $\mathrm{A}$ & G \\
\hline $\mathrm{C} / \mathrm{T}$ SNP & 167921 & $\mathrm{~T}$ & $\mathrm{~T}$ & $\mathrm{C}$ & $\mathrm{T}$ & $\mathrm{T}$ & $\mathrm{C}$ \\
\hline AHCY STR & 180274-99 & $(\mathrm{GT})_{13}$ & 170 & 168 & 168 & 166 & 174 \\
\hline$A g C A$ STR & & $(\mathrm{AC})_{21}$ & 123 & 125 & 127 & 125 & 127 \\
\hline
\end{tabular}

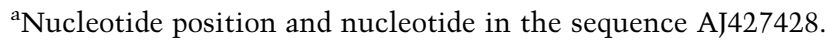


the manufactureŕs instructions. The reverse transcription into cDNA was performed by using $4 \mathrm{U}$ Omniscript (Qiagen), an oligo-dT primer $\left((\mathrm{T})_{24} \mathrm{~V}\right)$, and $10 \mu \mathrm{l}$ of the isolated RNA in a $20-\mu \mathrm{l}$ reaction.

A single assay was used for quantification of the entire ASIP gene transcript using a forward primer situated in exon 2 (5'-AAC CTG TTG GAT TTC CCT TCT G-3'), a reverse primer in exon $3\left(5^{\prime}\right.$-TTT CTG CTG ATC TTT TTG GAT TTC T-3'), and a TaqMan minor groove binding probe located at the boundary of exons 2 and 3 (ASIP34-probe FAM labeled: 5'-CTA TTG TGG CAC TGA AC-3'). Expression levels of the five different porcine ASIP transcripts were determined using a universal reverse primer located in exon 2 (5'-CAC AGG CAT ACC AGT AGG GTA GCT-3') in combination with exon-specific forward primers for ASIP-RALY (5'ATG CTC AGC ACC ACC CAG AT-3'), ASIP-1A (5'-CCA GAG AGG CCT CCC AGG-3'), ASIP-1A' (5'-GAA CCA TTG ATC TAA AAA CGA CAT CTT-3'), ASIP-1A1A' (5'-ATC CCA GAG AGA GAG CTG TTA TTG AC-3'), and ASIP-1B (5'-CAT CAA AGG CCT CCC AGG AT-3') and a universal TaqMan minor groove binding probe for each PCR located in exon 2 (ASIP2-probe FAM labeled: 5'-TCA CTC GCC TCC TCT T-3'). Porcine GAPDH transcript was determined as endogenous control using the forward (5'-CTG GTC ACC AGG GCT GCT T$\left.3^{\prime}\right)$ and reverse primer (5'-CTT GAC TGT GCC GTG GAA CT-3') in combination with a VIC-labeled TaqMan minor groove binding probe $\left(5^{\prime}\right.$-TCC ACT ACA TGG TCT ACA TGT-3'). The qRT-PCR was carried out with an ABI 7300 sequence detection system (Applied Biosystems, Darmstadt, Germany) in 20- $\mu \mathrm{l}$ reactions containing TaqMan Universal MasterMix (Applied Biosystems), $50 \mu \mathrm{M}$ forward, $50 \mu \mathrm{M}$ reverse primer, and $10-\mu \mathrm{M}$ TaqMan probe using an annealing and elongation temperature of $60^{\circ} \mathrm{C}$. The ASIP transcript-specific expression was normalized by division with the porcine GAPDH expression level $(\Delta C t)$ and the relative expression level was calculated by the $2^{-\Delta \Delta C t}$ method using the dorsal skin as calibrator. All assays were performed in triplicates.

\section{Results}

Resource family construction and linkage analysis. A Mangalitza $\times$ Piétrain resource family with $1492 \mathrm{~F}_{2}$ piglets was established. The Mangalitza $\times$ Piétrain $F_{1}$ animals showed a uniform gray phenotype (Fig. $1 B$ ). In the $F_{2}$ generation the color phenotypes were distributed as follows: 291 black-and-tan, 808 gray, and 393 black-spotted animals, respectively.
Sequence analyses of the MCR1 alleles (not shown) confirmed the homozygous $E^{+} / E^{+}$genotype in the Mangalitza founder boars. The parental Piétrain sows were $E^{P} / E^{P}$, except a single sow showing a novel allele termed $E^{P^{\prime}}$, where the ${ }^{121} \mathrm{~N}$ mutation was associated with ${ }^{163} \mathrm{~W}$ instead of ${ }^{163} \mathrm{R}$ as in the previously reported $E^{P}$ allele (Kijas et al. 1998b); the nucleotide sequence of this new $M C 1 R$ allele has been deposited in the EMBL nucleotide database under accession AM050718. The segregation of MCR1 alleles partially explained the variation in coat color phenotypes in the $\mathrm{F}_{2}$ generation since all black-spotted animals were homozygous $E^{P} / E^{P}$ like the Pietrain founders and all other $\mathrm{F}_{2}$ animals were homozygous or heterozygous for the wild type $E^{+}$ allele (Fig. 2). Assuming two independent segregating alleles at the MC1R locus and the ASIP locus, respectively, the observed phenotype distribution of black-and-tan $\left(a^{t} / a^{t}, E^{+} /-\right)$, gray $\left(A /-, E^{+} /-\right)$and black-spotted $\left(-/-, E^{P} / E^{P}\right)$ in the $\mathrm{F}_{2}$ population showed no significant deviation from the expected 3:4:9 proportion (chi-squared test, $p=0.264$ ).

Linkage mapping of the ASIP gene-associated microsatellite $A g C A$ showed significant linkage (LOD > 6.0) to markers ENDO and S0292, which have been previously mapped on the genetic linkage map (Ellegren et al. 1994), thus confirming its location on SSC17. In total five different $A g C A$ alleles were detected in the 166 genotyped animals of the Mangalitza $\times$ Piétrain resource population (Table 1). In 31 black-and-tan-colored $\mathrm{F}_{2}$ animals, both $A g C A$ marker alleles could be traced back, and in 29 animals, at least one of the $A g C A$ alleles was unequivocally of Mangalitza origin (91 informative meioses). There was no recombination between $A g C A$ and the coat color phenotype in the black-and$\tan$ animals. The estimated nonparametric $Z$ mean value was 16.2 and the LOD score value was 7.2.

Cloning and sequencing of porcine ASIP genomic and mRNA sequences. Five PAC and 11 BAC clones were assembled into a single genomic contig encompassing the entire sequence of ASIP and the downstream neighboring AHCY gene (Fig. 3). Two overlapping PAC clones from the German Landracederived TAIGP714 library were completely sequenced and the determined total genomic sequence of 204,138 bp was submitted to the EMBL database under accession No. AJ427478.

RACE PCR experiments revealed the existence of five different ASIP splice variants and the determined sequences were deposited into the EMBL nucleotide database (AJ634673-AJ634677). The different porcine ASIP transcripts had a common 3' end, including the entire open reading frame (ORF) 

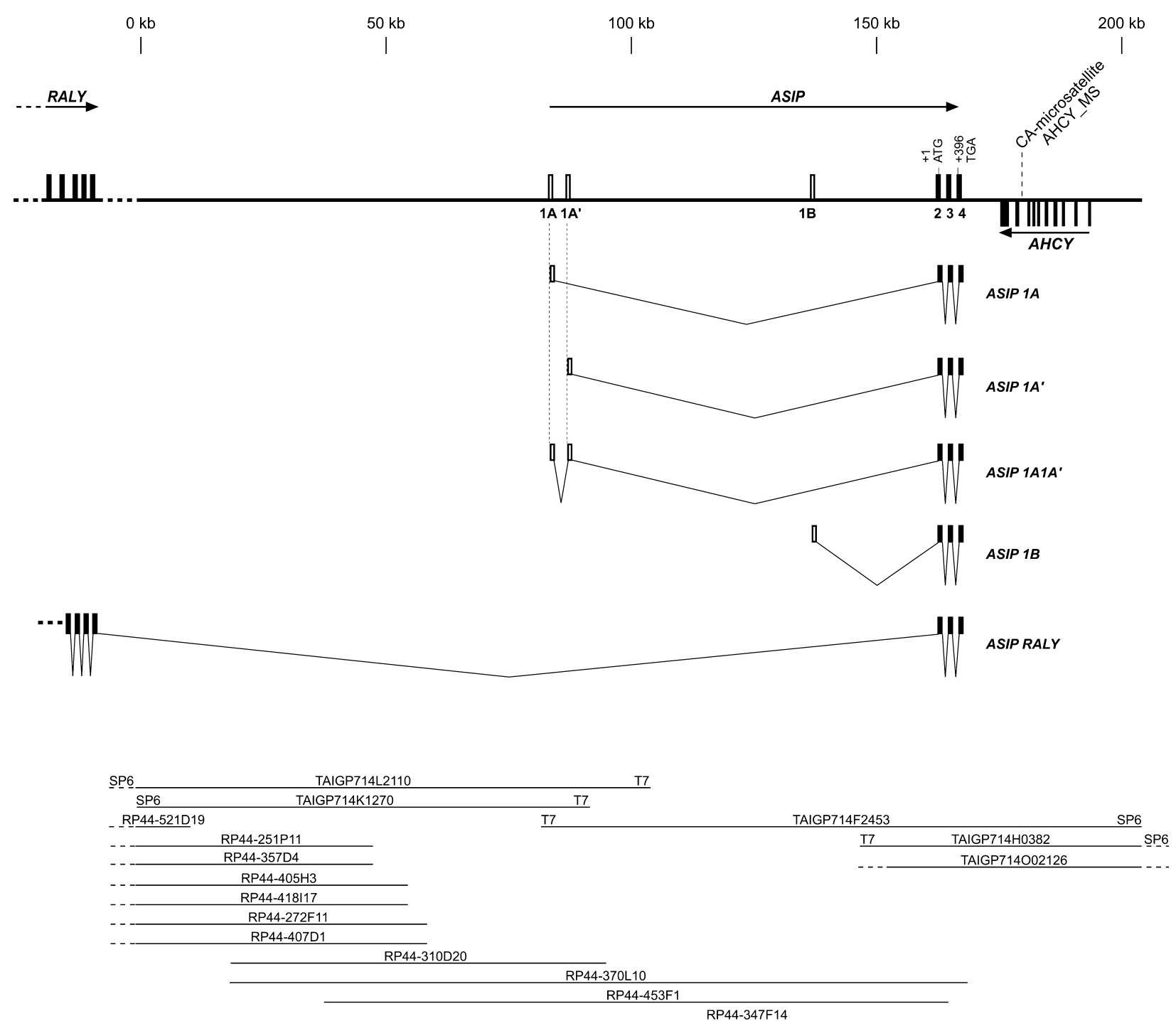

Fig. 3. Genomic structure of the porcine ASIP gene. Exons of the ASIP gene are shown as boxes. Untranslated regions are shown as open boxes, while protein-coding parts are shown in solid black. Exons are numbered according to the nomenclature of Bultman et al. (1992) and Chen et al. (1996). The coordinates at the top correspond to nucleotide positions in AJ427478. The five detected ASIP transcript variants are shown in the middle. The positions of the isolated PAC and BAC clones are represented by horizontal bars in the lower part.

for the 131-amino-acid-encompassing agouti signaling protein. Differences were observed in the $5^{\prime}$ ends of the different transcripts. Sequence comparison of the cDNA sequences with the genomic ASIP sequence led to the unequivocal annotation of the $5^{\prime}$ untranslated ASIP exons in the genomic sequence. We chose to adopt the numbering system of the murine Asip gene because this is the best studied mammalian ortholog (Fig. 3). Very similar to the murine Asip gene, the porcine ASIP gene contains two adjacent $5^{\prime}$ exons, termed exon $1 \mathrm{~A}$ and exon $1 \mathrm{~A}^{\prime}$, with high sequence conservation to the homologous mouse exons. Exons 1A and 1A' are located approximately $79 \mathrm{~kb}$ and approximately $77 \mathrm{~kb}$ upstream of exon 2 in the porcine ASIP gene, respectively. In the region of the so-called "hair-cycle-specific" exons 1B and $1 \mathrm{C}$, the porcine ASIP gene apparently had only one exon, which had moderate sequence conservation to the murine exon 1B. This exon was located about $25 \mathrm{~kb}$ upstream of exon 2. Four different porcine functional transcripts were initiated within the region of ASIP exons 1A to exon 1B. According to their exon content, these four transcripts were termed ASIP 1A, ASIP 1A', ASIP 1A1A', and ASIP $1 B$, respectively (Fig. 3). In addition to these four transcripts, a fifth potentially nonfunctional transcript termed ASIP RALY was consistently detected that arose from the fusion of exons from the upstream adjacent $R A L Y$ gene to the coding exons of the ASIP gene. In the ASIP RALY isoform the ASIP 


\section{Mangalitza}

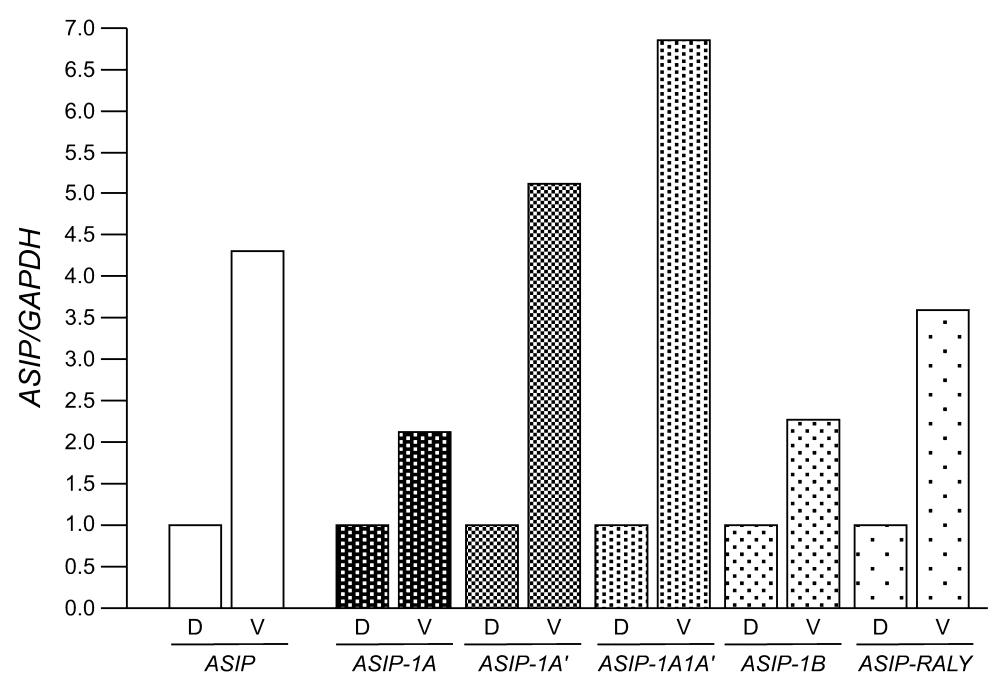

Hampshire

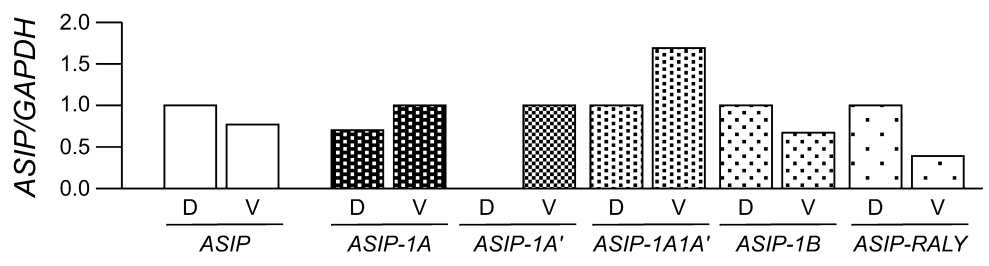

Fig. 4. Expression levels of ASIP in ventral (V) and dorsal (D) skin of swallow-bellied Mangalitza pigs and a black Hampshire pig. Columns show relative expression levels of ASIP normalized to porcine GAPDH analyzed by qRT-PCR for ASIP in total (left) and for the five different splice variants (right). Note that the expression levels of all ASIP splice forms are considerably higher in the yellow pigmented ventral skin of the Mangalitza pig compared with the black pigmented dorsal skin of the Mangalitza or the uniformly black skin of the Hampshire pig.
ORF is probably not translated because it is preceded by the large $R A L Y$ ORF.

The transcription initiation sites of the porcine ASIP gene are not associated with CpG islands. There were also no unambiguous TATA box motifs in the correct distance to the observed transcription start sites. Thirty nucleotides upstream of exon 1B, the sequence motif TTAATAA could potentially resemble a variant TATA box motif, however, no TATA box-like sequences were identified upstream of exon 1A and exon 1A'. The entire sequenced contig of 204,138 bp has an average GC content of $41 \%$, which corresponds to the average of mammalian genomes. The sequenced region has a high content of repetitive elements $(56 \%)$. This was largely a result of a rather high content of long interspersed element (LINE) sequences (23.8\%). The presence of many full-length LINE sequences greatly hampered a systematic mutation analysis of the potentially regulatory first intron because some regions could not be PCR-amplified because of the high repeat content.

Polymorphism screening. Coding and noncoding exons were screened for polymorphisms to identify potential sequence variation that could explain phenotypic differences among the observed coat color phenotypes. Between Mangalitza and
Piétrain pigs we could not detect any differences that were consistently associated with the coat color phenotype. Several cosegregating, noncoding, intergenic and intronic nucleotide polymorphisms (21 SNPs, 2 indels, 1 microsatellite) were detected in a mutation scan of a three-generation subfamily of the resource population (Table 1). Nonparametric linkage analysis within this family confirmed the complete linkage ( $Z$ mean value of 9.5$)$ of two distinct haplotypes termed $a^{t}-1$ and $a^{t}-2$ (Table 1) cosegregating with the Mangalitza phenotype (Fig. 2).

qRT-PCR experiments. In Mangalitza the relative porcine ASIP expression level differed widely between the ventral and dorsal skin, but every transcript could be detected in both regions. In general, the ventral yellow skin showed a 4.3-fold higher gene expression than the dorsal black skin (Fig. 4). The highest relative mRNA expression difference between ventral and dorsal was encountered for the $A S I P-1 A^{\prime}$ and $A S I P-1 A 1 A^{\prime}$ transcripts, followed by $A S I P-R A L Y$. For the $A S I P-1 A$ and $A S I P-1 B$ transcripts only an approximately twofold difference in expression levels was observed between the two differently pigmented skin regions. In a Hampshire sow, assumed to be homozygous for the wild-type $A S I P^{*} A$ allele, we detected only small differences in $A S I P$ expression level between the ventral and dorsal 
skin (Fig. 4). Furthermore, the relative expression level of $A S I P$, in relation to $G A P D H$, was significantly higher in the ventral skin of Mangalitza pigs compared with this Hampshire sow. The ASIP-1A' transcript could not be detected in the dorsal skin at all and only the $A S I P-1 A 1 A^{\prime}$ variant was expressed nearly twice as high in the ventral skin compared with the dorsal region.

\section{Discussion}

Expression studies in black-and-tan mice indicate that the level of Asip mRNA correlates with the synthesis of yellow pigment (Bultman et al. 1992), but it is still not clear why Asip gene expression should be restricted to the ventrum of $a^{t} / a^{t}$ mice. Recently, the molecular analysis of the droopy ear mouse mutant, which also produces a dorsal-to-ventral transformation of flank coat color by allowing expansion of the ventral-specific Asip transcript, revealed a loss of function mutation in the Tbx15 gene (Candille et al. 2004). Early embryonic expression of Tbx15 in dorsal mesenchyme is complementary to Asip expression in ventral mesenchyme; in the absence of Tbx15, expression of Asip in both embryos and postnatal animals is displaced dorsally. Agouti protein has a short radius of action (Silvers and Russel 1955) and can be switched on and off during a single hair cycle (Bultman et al. 1992, 1994; Vrieling et al. 1994); thus, its regulated expression is thought to be responsible for the cream-colored or yellow ventral surface of mice carrying the black-and-tan allele.

We chose swallow-bellied Mangalitza pigs as a similar model for further examination of this special coat color phenotype in mammals because the phenotype of these pigs corresponds closely to $a^{t} / a^{t}$ and to a lesser extent to $A^{W} / A^{W}$ mice, respectively. The segregation pattern of the MCR1 and ASIP alleles in the established porcine $\mathrm{F}_{2}$ resource population and the observed complete linkage of $A S I P$-associated alleles confirmed the hypothesis that the color pattern observed in the Mangalitza is most likely caused by a recessive allele at the porcine ASIP gene, probably corresponding to the $a^{t}$ allele in black-and-tan mice. Coding nucleotide ASIP polymorphisms could be excluded as the causative mutation for the $a^{t}$ allele. Although a variety of noncoding DNA polymorphisms in the ASIP gene region showed complete linkage to the inheritance of the black-and-tan phenotype, they could also be excluded as causative mutations because two different $a^{t}$ cosegregating haplotypes occurred in the two founder Mangalitza boars (Fig. 2). This can be explained by the following three possibilities: (1) they do not share the same causative mutation, (2) the causative mutation is lo- cated outside the sequenced region, or (3) the IBD (identical by descent) region is rather small and not covered by the sequenced regions.

Taking into account that there is a remarkable ASIP expression difference between the black dorsal skin and the yellow ventral skin in comparison to the nearly uniform expression level in the examined Hampshire pig, which presumably is homozygous for ASIP wild-type alleles, it seems likely that the observed differences in dorsal and ventral pigmentation result from this specific expression pattern of ASIP. The five detected alternative ASIP transcripts expressed in porcine skin contain different sets of $5^{\prime}$ untranslated exons and are consistent with the observed molecular heterogeneity among the $5^{\prime}$ ends in mice (Bultman et al. 1994) and cattle (Girardot et al. 2005). Two predominant Asip mRNA transcripts that differ by virtue of their transcriptional initiation site and $5^{\prime}$-untranslated exons have been identified in mice. A "hair-cycle-specific" transcript is expressed in both dorsal and ventral skin for 2-3 days during early hair growth, while a "ventral-specific" transcript is expressed throughout the entire period of active hair growth, but only in ventral skin (Bultman et al. 1994; Vrieling et al. 1994; Chen et al. 1996). As reported in black-and-tan mice, we have also observed increased expression of ventral-specific transcripts containing the exons $1 \mathrm{~A}$ and $1 \mathrm{~A}^{\prime}$ in Mangalitza pigs. Mice carrying the $a^{t}$ allele express only the ventralspecific Asip transcript (Bultman et al. 1994; Vrieling et al. 1994) and have black dorsal hairs and yellow ventral hairs, with a sharp boundary. Ventral-specific Asip isoforms are also expressed in developing skin from embryonic day 10.5 and beyond and may play a role in pigment cell differentiation (Millar et al. 1995). Thus, regulatory elements for ventral-specific Agouti isoforms are responsive to dorsoventral positional cues established in the embryo and whose effects persist after birth. Therefore, our data provide evidence for the existence of a potentially ventral-specific regulatory ASIP mutation in swallow-bellied Mangalitza pigs similar to the murine $a^{t}$ mutation.

\section{Acknowledgments}

The authors thank D. Seinige, H. Klippert-Hasberg, S. Neander, and B. Hayn for expert technical assistance, the staff of the Experimental Station Thalhausen of the Technical University of Munich for careful animal husbandry, and D. Fries for the color phenotyping of $\mathrm{F}_{2}$ animals. They also thank P. de Jong and his lab for providing the RPCI-44 library and excellent supplementary information. This study was supported by a grant of the German Research Council DFG (Le 1032/12-1) to TL. 


\section{References}

1. Abecasis GR, Cherny SS, Cookson WO, Cardon LR (2002) Merlin-rapid analysis of dense genetic maps using sparse gene flow trees. Nature Genet 30, 97-101

2. Al-Bayati H, Duscher S, Kollers S, Rettenberger G, Fries R, et al. (1999) Construction and characterization of a porcine P1-derived artificial chromosome (PAC) library covering 3.2 genome equivalents and cytogenetical assignment of six type I and type II loci. Mamm Genome 10, 569-572

3. Bultman SJ, Michaud EJ, Woychik RP (1992) Molecular characterization of the mouse agouti locus. Cell 71, 1195-1204

4. Bultman SJ, Klebig ML, Michaud EJ, Sweet HO, Davisson MT, et al. (1994) Molecular analysis of reverse mutations from nonagouti $(a)$ to black-and-tan $\left(a^{t}\right)$ and white-bellied agouti $\left(A^{w}\right)$ reveals alternative forms of agouti transcripts. Genes Dev 8, 481-490

5. Candille SI, Van Raamsdonk CD, Chen C, Kuijper S, Chen-Tsai Y, et al. (2004) Dorsoventral patterning of the mouse coat by Tbx15. PLoS Biol 2, E3

6. Carroll L, Voisey J, van Daal A (2004) Mouse models of obesity. Clin Dermatol 22, 345-349

7. Chen Y, Duhl DM, Barsh GS (1996) Opposite orientations of an inverted duplication and allelic variation at the mouse agouti locus. Genetics 144, 265-277

8. Ellegren $\mathrm{H}$, Chowdhary BP, Johansson M, Marklund L, Fredholm M, et al. (1994) A primary linkage map of the porcine genome reveals a low rate of genetic recombination. Genetics 137, 1089-1100

9. Girardot M, Martin J, Guibert S, Leveziel H, Julien R, et al. (2005) Widespread expression of the bovine Agouti gene results from at least three alternative promoters. Pigment Cell Res 18, 34-41

10. Harris RB, Mitchell TD, Mynatt RL (2002) Leptin responsiveness in mice that ectopically express agouti protein. Physiol Behav 75, 159-167

11. Huszar D, Lynch CA, Fairchild-Huntress V, Dunmore JH, Fang Q, et al. (1997) Targeted disruption of the melanocortin-4 receptor results in obesity in mice. Cell 88: $131-141$

12. Kerns JA, Newton J, Berryere TG, Rubin EM, Cheng JF, et al. (2004) Characterization of the dog Agouti gene and a nonagoutimutation in German Shepherd Dogs. Mamm Genome 15, 798-808

13. Kijas JMH, Törnsten A, Chowdhary B, Andersson L (1998a) Porcine agouti gene map position SSC 17q21. Chromosome Res 6, 243

14. Kijas JMH, Wales R, Törnsten A, Chardon P, Moller $\mathrm{M}$, et al. (1998b) Melanocortin receptor 1 (MC1R) mutations and coat color in pigs. Genetics 150, $1177-1185$

15. Kijas JMH, Moller M, Plastow G, Andersson L (2001) A frameshift mutation in $M C 1 R$ and a high frequency of somatic reversions cause black spotting in pigs. Genetics 158, 779-785

16. Kwon HY, Bultman SJ, Loffler C, Chen WJ, Furdon PJ, et al. (1994) Molecular structure and chromosomal mapping of the human homolog of the agouti gene. Proc Natl Acad Sci USA 91, 9760-9764

17. Leeb T, Rohrer GA (2002) Characterization and chromosome assignment of the porcine AHCY gene for Sadenosylhomocysteine hydrolase. Cytogenet Genome Res 97, 116-119

18. Leeb T, Deppe A, Kriegesmann B, Brenig B (2000) Genomic structure and nucleotide polymorphisms of the porcine agouti signalling protein gene $(A S I P)$. Anim Genet 31, 335-336

19. Lu D, Willard D, Patel IR, Kadwell S, Overton L, et al. (1994) Agouti protein is an antagonist of the melanocyte-stimulating-hormone receptor. Nature 371, 799-802

20. Martins-Wess F, Voß-Nemitz R, Drögemüller C, Brenig B, Leeb T (2002) Construction of a 1.2-Mb BAC/ PAC Contig of the porcine gene RYR1 Region on SSC 6q1.2 and comparative analysis with HSA 19q13.13. Genomics 80: 416-422

21. Millar SE, Miller MW, Stevens ME, Barsh GS (1995) Expression and transgenic studies of the mouse agouti gene provide insight into the mechanisms by which mammalian coat color patterns are generated. Development $121,3223-3232$

22. Silvers WK, Russel ES (1955) An experimental approach to action of genes at the agouti locus in mouse. J Exp Zool 130, 199-220

23. Siracusa LD (1994) The agouti gene: turned on to yellow. Trends Genet 10 , 423-428

24. Vage DI, Lu D, Klungland H, Lien S, Adalsteinsson S, et al. (1997) A non-epistatic interaction of agouti and extension in the fox, Vulpes vulpes. Nat Genet 15, $311-315$

25. Vrieling H, Duhl DM, Millar SE, Miller KA, Barsh GS (1994) Differences in dorsal and ventral pigmentation result from regional expression of the mouse agouti gene. Proc Natl Acad Sci U S A 91, $5667-5671$

26. Wiedemann S, Kijas J, Andersson L, Fries R (2000) Genetic mapping of agouti in a Mangalitza x Piétrain cross. Proceedings of the 27 th International Conference on Animal Genetics, Minnesota, July 22-26, 2000 , B060, 37 